\title{
Resiliencia Socioecológica: aportaciones y retos desde la Antropología
}

\section{Socioecological Resilience: contributions and challenges from Anthropology}

\author{
Javier ESCALERA REYES \\ Esteban RUIZ BALLESTEROS \\ GISAP. Departamento de Ciencias Sociales. Universidad Pablo de Olavide (Sevilla) \\ fjescrey@upo.es \\ eruibal@upo.es
}

Recibido: 7 de febrero de 2011

Aceptado: 14 de marzo de 2011

\begin{abstract}
Resumen
La necesidad de una comprensión compleja de los territorios y su devenir está siendo encarada a través de nociones como las de sistema complejo adaptativo y socio-ecosistema. La sensibilidad ambiental es cada vez más una sensibilidad socio-ambiental. Así la sostenibilidad ha comenzado a reformularse desde las propias ciencias de la naturaleza a través del concepto de resiliencia socio-ecológica, reclamándose una transdisciplinaridad que sea capaz de articular operativamente las dimensiones naturales y culturales del entorno. El concurso de la Antropología Social en este proceso es capital en tanto en cuanto puede aportar una perspectiva sólida en torno a: (1) el desarrollo teórico-metodológico de las nociones de socioecosistema y resiliencia socio-ecológica; (2) el debate en torno a la articulación entre cultura y naturaleza; y (3) la apuesta etnográfica como metodología especialmente apropiada para comprender la realidad socio-natural. El texto avanza sumariamente en esta reflexión teórica-metodológica en virtud de nuestras recientes investigaciones en Ecuador y Costa Rica.
\end{abstract}

Palabras clave: resiliencia socio-ecológica, socioecosistema, medio ambiente, sostenibilidad.

\begin{abstract}
The need of a complex understanding of the territories and its evolution is being addressed through notions such as adaptive complex system and socio-ecosystem. Environmental sensitivity is increasingly a socio-environmental sensitivity. So sustainability has begun to be reformulated from the Nature Sciences through the concept of socio-ecological resilience, claiming a transdisciplinarity that is capable of operationally articulate the natural and cultural dimensions of the environment. The participation of Social Anthropology in this process is key insofar it can provide a solid perspective on: (1) the development of notions as socioecosystem and socio-ecological resilience; (2) the debate on the relationship between culture and nature; and (3) the ethnography as an especially appropriate methodology to understand socio-natural reality. The text goes forward in this theoretical and methodological reflection through our recent research in Ecuador and Costa Rica.
\end{abstract}


Keywords: socio-ecological resilience, socioecosystem, environment, sustainability.

Referencia normalizada: Escalera Reyes, J. \& Ruiz Ballesteros, E. (2011). Resiliencia Socioecológica: aportaciones y retos desde la Antropología. Revista de Antropología Social, 20, 109-135.

SUMARIO: 1. Introducción. 2. Ecología más allá del "equilibrio" y socioecosistemas. 3. Antropología, ecosistema y resiliencia. 4. Resiliencia y sostenibilidad. 5. Operativizar el estudio de la resiliencia socio-ecológica. 6. ¿Puede el turismo ser una estrategia resiliente? Reflexiones desde Agua Blanca. 7. Coopesilencio: comunitarización y resiliencia. 8. Resiliencia y Antropología Ambiental. 9. Referencias bibliográficas.

\section{Introducción}

... to better understand ecosystems we need to understand the dominant organism on this planet"(Abel y Stepp, 2003:9).

...understanding of environment as both the product of and the setting for human interactions, which link dynamic structural analyses of environmental processes with an appreciation of human agency in environmental transformation... (Scoones, 1999: 479).

Está comúnmente asumido que la dimensión socio-cultural no puede desvincularse del concepto ecosistema. Sin embargo, todavía existe una enorme falla entre el discurso y la práctica. A pesar de los artículos científicos, las declaraciones, las directivas y las resoluciones de todo tipo que afirman y promueven la perspectiva socio-ecológica para la conservación de espacios naturales y la biodiversidad, el desarrollo sostenible o la lucha contra el cambio global, la intervención ambiental aún se basa fundamentalmente en un enfoque biofísico que, ya sea por inercia o por falta de confianza, da primacía absoluta a aspectos y elementos "naturales", relegando a un segundo plano "lo social". Difícilmente una intervención ambiental alcanzará sus objetivos si no se tienen en cuenta las dimensiones biofísicas y socioculturales simultáneamente y con la misma profundidad, estableciendo estrategias y mecanismos que lo hagan factible. Para ello, es esencial un diálogo amplio y profundo entre los científicos biofísicos y sociales a través del cual se pueda establecer un lenguaje compartido e instrumentos comunes de análisis. Pero a pesar de la voluntad y la predisposición de un número creciente de miembros de ambas comunidades científicas, esto no parece tarea fácil.

Scoones (1999), al tiempo que señala el amplio espacio abierto por la "nueva ecología" para la inter y la transdisciplinaridad, pone de relieve la escasa materialización de este potencial. Más recientemente, Abel y Stepp (2003) redundan en la misma apreciación, invitando a la antropología a complementar los pasos que se están dando desde ciertas corrientes ecológicas, que reclaman vivamente la integración de la perspectiva socio-cultural en la investigación ambiental. 
Nuestro trabajo se enmarca en esta tendencia, en el convencimiento de que, más que nunca, sin un concurso intenso de la Antropología no podrá culminarse una propuesta cabal de conocimiento ambiental. Nos centramos en el concepto resiliencia socio-ecológica porque en él toma cuerpo una consideración integral —biofísica y sociocultural - de lo ambiental, que desde un enfoque complejo puede hacer posible una operativización analítica de la sostenibilidad.

La resiliencia socio-ecológica se puede entender como la capacidad de un socioecosistema sujeto a algún tipo de stress —en el sentido más básico del término- o de cambio profundo - no necesariamente negativo - para regenerarse a sí mismo sin alterar sustancialmente su forma y funciones, en una especie de "conservación creativa". En esta tensión permanente de lo vivo entre estabilidad y cambio, la resiliencia es una noción que nos permite pensar de una manera particular la relación entre naturaleza y sociedad. Nos invita a una perspectiva que antepone el proceso a la forma, el cambio constante al equilibrio, y la relación dinámica a la articulación estática. La resiliencia nos ayuda a cambiar la perspectiva de análisis desde modelos simples de relación causa-efecto, a sistemas complejos y relaciones no lineales, siempre considerando la dimensión escalar del tiempo y el espacio (Davidson-Hunt y Berkes, 2003:76).

Es indiscutible el peso de lo humano en este tipo de procesos y, por tanto, el papel potencial de la Antropología en el desarrollo del concepto "resiliencia socioecológica" que puede llevarnos a una mejor comprensión de la dimensión medioambiental y, por consiguiente, al diseño más acertado de estrategias de intervención. Como veremos más adelante, el propio concepto no es del todo ajeno a la Antropología (Vayda y McCay, 1975).

\section{Ecología más allá del "equilibrio" y socioecosistemas}

La ecología y el pensamiento occidental sobre la naturaleza se han construido principalmente sobre el "equilibrium thinking" (Scoones, 1999), lo que propicia enfoques eminentemente estáticos y esencialistas. Si bien se asume que la sociedad cambia, hay un planteamiento implícito de que la naturaleza es lo que permanece y que, si ésta cambia, lo hace en negativo y fundamentalmente por efecto antrópico. Por eso la protección ambiental es para muchos sinónimo de ausencia humana. Es en este contexto donde debemos encuadrar conceptualizaciones tan importantes para la gestión ambiental como la de "capacidad de carga", vinculada a un supuesto clímax de estabilidad. O explicar la visión estática de la naturaleza, que ha prevalecido y prevalece tanto en la militancia ecologista como en las esferas de planificación, diseño y gestión ambiental, e incluso en las ciencias sociales en general y la antropología en particular (Scoones, 1999). Sin embargo, este tipo de visiones comenzaron a ser cuestionadas, a partir de los pasados años 70, con la evidencia de las propiedades inestables tanto de los modelos como de los ecosistemas reales y el desarrollo de las matemáticas ecológicas. En esos momentos emergen enfoques ecológicos que tienen su anclaje en principios como la inestabilidad, la variación, la complejidad y la incertidumbre, y que se plasman en conceptualizaciones como 
la de los sistemas no lineales, la dinámica caótica, la sensibilidad a condiciones iniciales, la impredecibilidad o los sistemas estocásticos.

Surge así un lenguaje nuevo para tratar el comportamiento de los ecosistemas, una sensibilidad que se va aplicando con éxito a distintos contextos de investigación - bosques, lagos, arrecifes de coral...- y con resultados positivos en intervenciones ambientales. Sin embargo, las Ciencias Sociales, a pesar de su evidente interés por lo ambiental, han seguido mayoritariamente ligadas a una visión donde prima lo estático y sobre todo el equilibrio cuando pensamos el medio ambiente: el entorno como algo integrado y regulado, y su cambio como proceso lineal, estable y predecible; la naturaleza marcada por el balance. Desde la antropología ecológica o la etnoecología se ha argumentado insistente y acertadamente sobre la estrecha interacción naturaleza-sociedad como un todo funcional (Rappaport, 1967, 1968), pero desde esta perspectiva no se ha interrogado por las complejidades de la dinámica social o ecológica, propiciándose -implícita o explícitamente- una visión estática del medio y del conocimiento sobre él (Scoones, 1999: 485). Se establece así una doble consideración muy simplificadora: los humanos y el medio o bien están en una relación de armonía - ligada a territorios ocupados por "culturas ancestrales" o "sociedades tradicionales" - o de desarmonía — ligada directamente a la irrupción de la lógica capitalista en la cultura- - La sostenibilidad se entiende, por tanto, como sinónimo de equilibrio y el objetivo no será otro que una quimérica tendencia al "balance". De ahí la dificultad para hacer que este concepto pueda adquirir operatividad analítica.

Sin embargo, Holling (1973) comenzó hace cuatro décadas a hacer evidente la complejidad de los fenómenos ecológicos en sí mismos, poniendo el acento en el cambio antes que en la estabilidad. Sentó así las bases de la resiliencia como perspectiva de análisis. Para comprender el funcionamiento de los ecosistemas, nos propone que consideremos también el azar. Los ecosistemas, en constante transformación, se organizan en torno a ámbitos de atracción y umbrales ${ }^{1}$ que, si se sobrepasan, implican cambios sustanciales en el propio ecosistema. El análisis sistémico se ha fundamentado tradicionalmente en el equilibrio por mera comodidad analítica; pero es la flexibilidad el concepto clave para entender cabalmente un sistema ecológico.

Sus propuestas y conclusiones pusieron en entredicho el "sentido común" del pensamiento ecológico clásico, hasta proclamar que la estabilidad es un problema en sí mismo: si el medio es homogéneo en el espacio y en el tiempo tendrá pocas fluctuaciones y, por tanto, baja resiliencia: su riesgo de colapso será mayor (Holling, 1973:18). Asimismo, la inestabilidad numérica de una especie puede propiciar la resiliencia del conjunto del sistema, ya que facilita la mayor diversidad de especies y la modularidad espacial (Holling, 1973:19). A la vista está que el principal aporte de este autor fue la integración de fenómenos que se entienden como contradictorios: flexibilidad y perdurabilidad a un tiempo, variabilidad en número y persistencia, eventos azarosos y factores deterministas.

\footnotetext{
${ }^{1}$ Para una aproximación general a estos conceptos, ver Scheffer, 2009.
} 
A Holling $(1973,2001)$ le debemos ideas muy fértiles para comprender los socio-ecosistemas: que los sistemas inventan y experimentan; que los cambios no son graduales ni ordenados, sino espasmódicos, seguidos de un tiempo de reinvención y desarrollo ${ }^{2}$; o que un sistema muy sólido y rígido que no permita siquiera la creatividad será sólo en apariencia resiliente, ya que, si bien resiste cambios y condicionantes exteriores, no permite la creatividad interna por excesivo control y ahogo, y, por tanto, porta la semilla de su propio colapso.

De esta manera, el concepto de sistema que lleva implícita la idea de ecosistema va transformándose hacia una noción mucho más desarrollada de "sistema adaptativo complejo", que satisface con mayor garantía la necesidad de estudiar el socioecosistema. Esta conceptualización implica la consideración de la no linealidad, sin retroalimentación simple, con bifurcaciones, saltos y discontinuidades tempoespaciales, por consiguiente, de escasa posibilidad de predicción pero sin caer en el puro azar.

Por su parte, y en coherencia con todo ello, la noción de socioecosistema es un medio para superar las fragmentaciones epistemológicas, ontológicas y analíticas que nos atenazan impidiendo una consideración sistémica compleja que articule lo socio-cultural y lo biofisico de forma operativa. El socioecosistema es un sistema - comunidades humanas que ocupan y se relacionan con un territorio y viceversacaracterizado por una densa red de interrelaciones entre las dimensiones sociocultural y ecológica, autoorganizados, no lineales y sometidos a la incertidumbre (Folke et al., 2002; Holling, 2001). Sin embargo, es conveniente tener en cuenta que los socioecosistemas son unidades de análisis elegidas por el investigador en virtud de un interés especifico (Ostrom, 2009), deben entenderse como sistemas abiertos sólo comprensibles globalmente desde la metáfora de la panarquía (Holling, 2001), en una estructura anidada de sistema de sistemas, con afecciones múltiples a distintas escalas y velocidades (Holling, 2001).

Esta consideración de los socioecosistemas nos lleva irremisiblemente a la idea de coevolucion (Noorgard, 1984), ya que es una encarnación concreta de la misma. En el seno de los sistemas socio-ecológicos debería asumirse el protagonismo humano, la difuminación de la dicotomía naturaleza-cultura (Ingold, 2000), y asimismo las relaciones de poder, la libertad, la dependencia y la autonomía de los grupos humanos. Todo ello hace muy difícil comprender en toda su extensión cualquier socioecosistema, aunque no cabe duda de la oportunidad y potencialidad de la investigación antropológica para hacerlo operativo. A pesar de que la antropología ambiental no haya asumido una visión tan dinámica y compleja del medio, es probablemente la ciencia social que más ha trabajado por comprender los resortes materiales y simbólicos que relacionan a los entornos y sus habitantes humanos.

\section{Antropología, ecosistema y resiliencia}

La relación entre sociedad y naturaleza es una de las preocupaciones clásicas en Antropología. La tensión entre determinismo ambiental y posibilismo histórico

\footnotetext{
${ }^{2}$ Ver su propuesta de dinámica de los sistemas en torno a la noción de Panarchy (Holling 2001).
} 
desembocó en la ecología cultural de Stewart (1955), con un tratamiento particular de las nociones de adaptación y cambio. Davidson-Hunt y Berkes (2003) señalan a Geertz (1963) como introductor de la idea de ecosistema en la Antropología. Desde entonces este concepto ha tenido un amplio recorrido en nuestra disciplina, pero casi siempre anclado a un acusado matiz de estabilidad, adaptación y ritualismo. Estas circunstancias se han querido ver espacialmente reflejadas en la obra de Rappaport (1968), pero quedaron en cierto sentido reformuladas en trabajos posteriores del autor (Rappaport, 1990). Moran (1990) nos ofrece un profundo repaso de la importancia del concepto ecosistema para la antropología, reconociendo su valía a pesar de la necesidad de profundizar y flexibilizar su uso, alejándolo del sentido de equilibrio que lo hipoteca.

Es precisamente en este sentido en el que debemos entender la primera utilización del concepto resiliencia en Antropología. Para Vayda y McCay (1975), la cultura no podía ser considerada como un factor de equilibrio ecológico, tal como argüía Rappaport. Este uso de cultura supone, implícitamente, dotarla de un sentido de resiliencia similar al que se aplica en el ámbito de la ingeniería: recuperación de un sistema para volver a la misma configuración antes de la perturbación. Vayda y McCay apuntan que lo que Rappaport entiende como mecanismo de estabilidad - la cultura - debe ser entendido mejor como mecanismo de resiliencia, que permitiría cambiar encarando el azar y los retos ambientales. Resiliencia no implica conservación, ni estabilidad, sino paradójicamente cambio.

Es, por tanto, el concepto de resiliencia el que termina dotando de dinámica a la antropología ecológica. Davidson-Hunt y Berkes (2003: 61 y ss.) señalan que el uso de la resiliencia en antropología ha venido sirviendo para explicar distintos tipos de fenómenos socio-ambientales: estrategias generalistas o especializadas en la consecución de recursos; incertidumbre y sorpresa en la gestión de recursos; capacidad de adaptación y grado de centralización en el uso y gestión de recursos.

Sin embargo, para operativizar el concepto de resiliencia en el campo ambiental, es necesario ir más allá de las dicotomías cultura/naturaleza e individuo/cultura (Davidson y Berkes, 2003: 68 y ss.). Es aquí donde encuentran eco las reformulaciones de la relación naturaleza-sociedad-cultura que han marcado la antropología de finales del siglo XX (Descola, 2005; Descola y Pálsson, 1996; Escobar, 1998, 1999; Ingold, 2000; Latour, 1991; Pálsson, 1996; Raffestin, 1996; Wilson, 1992). El nuevo uso del concepto resiliencia debe enmarcarse precisamente en este contexto de cuestionamiento de las categorías tradicionales del pensamiento occidental, que dan pie a una auténtica integración analítica humanos/entorno, sea a través del concepto dwelling de Ingold (2000), o del concepto human-in-nature (Davidson-Hunt y Berkes, 2003). Desde esta perspectiva, nos parece especialmente apropiado el uso del concepto resiliencia socio-ecológica en la investigación antropológica.

\section{Resiliencia y sostenibilidad}

Sea como una teoría, en el sentido fuerte del término, o como una metáfora, la idea de resiliencia socio-ecológica puede utilizarse como una matriz para hacer efectiva la perspectiva socio-ecológica. Si es el cambio y no la estabilidad lo que 
caracteriza a los sistemas socio-ecológicos; entonces la conservación y la sostenibilidad deben convertirse inevitablemente en dinámicos.

La resiliencia de un socio-ecosistema debe ser entendida como su capacidad para hacer frente a los cambios, no para resistirse a ellos — pues son inevitables-. Se trata, por tanto, de la habilidad de un socioecosistema para absorber creativamente la trasformación sin perder su identidad como tal. Una débil resiliencia da paso a la vulnerabilidad de los socioecosistemas, que verían comprometida su continuidad ante las transformaciones externas o internas que no son capaces de integrar o asimilar. La resiliencia es un concepto paradójico, ya que refiere a la capacidad de un sistema de no cambiar sustancialmente aan cuando el cambio es su atributo permanente.

Sin embargo, esa capacidad de cambio puede ser deseada o indeseada desde el punto de vista humano. Si queremos que el sistema siga siendo como es, nos interesara alimentar su resiliencia para que permanezca, pero si, por el contrario, el sistema nos resulta indeseable, buscaremos la forma de superar su resiliencia para que se transforme. En un socioecosistema, esta circunstancia es precisamente más evidente desde la perspectiva sociocultural que desde un enfoque biofísico. De este modo, debe quedar claro que la resiliencia es una herramienta para evaluar las posibilidades de transformación de un socioecosistema, pero nunca un valor en sí mismo: ser resiliente puede resultar positivo o negativo, según nuestra valoración global del sistema que desarrolla esa capacidad.

La resiliencia, por tanto, es la medida de las posibilidades de cambio sustancial de un sistema, de su transformación y de los factores - externos e internos-que la animan; se trata pues de una estrategia de análisis. Resiliencia no es lo mismo que sostenibilidad, la resiliencia es un atributo del sistema en sí del que no se puede deducir deseabilidad o positividad. La sostenibilidad parte del análisis de la deseabilidad del estado del socioecosistema: tanto en sus aspectos biofísicos como socioculturales. Un sistema puede ser resiliente pero no deseado, entonces el objetivo sería cambiarlo.

El estado resiliente de un socioecosistema depende de multitud de factores interdependientes, por eso no parece lógico trazar un retrato robot del "socioecosistema resiliente", máxime cuando se invita cada vez más a pensar que la resiliencia tiene un innegable carácter contextual, de caso. No obstante, conviene hacerse una idea más global del sentido de la resiliencia. Tal como hemos propuesto en otro lugar (Ruiz-Ballesteros, 2011), la resiliencia es una actitud antes que un estado, una forma procesual antes que un contenido, una manera de modular-modelar la dinámica inherente a un socioecosistema, sólo entendible desde el protagonismo humano correspondiente.

Como vemos, nos situamos muy lejos de las visiones simplificadoras que harían de la resiliencia una forma de resistencia o de adaptación ${ }^{3}$ mecánica, más bien estamos refiriéndonos a esa capacidad de un sistema para absorber la perturbación y reorganizarse, al tiempo que experimenta el cambio reteniendo esencialmente

\footnotetext{
${ }^{3}$ Para un concepto complejo y sobre todo creativo de adaptación, ver Bergson (2008).
} 
las mismas funciones, estructura, identidad y retroalimentaciones (Walker y Salt, 2006). Aquí entran en juego la creatividad, el carácter proactivo y la innovación. Es precisamente la innovación la que permite mantener funciones, estructura e identidad, a pesar del cambio incesante e inherente a todo socioecosistema. Aprendizaje, reorganización, innovación..., todos estos atributos tienen una especial incidencia desde la dimensión social. La vinculación del concepto innovación a la resiliencia nos parece capital, ya que hace que los socioecosistemas no sean agentes pasivos, sino antes bien proactivos en su propia configuración. Por todo ello, la capacidad resiliente de los sistemas complejos adaptativos se podrían focalizar en tres rasgos generales (Carpenter et alt., 2001; Turner et alt, 2003):

- La capacidad de lidiar con el cambio manteniendo función y estructura; es decir, de "seguir siendo" a pesar de las corrientes y flujos que lo impedirían.

- La habilidad de reorganizarse, relacionada especialmente con la capacidad de reorganización endógena más que con la forzada externamente — ecosistema evolutivo y redes sociales-.

- La capacidad adaptativa, que se relaciona con mecanismos para la evolución de la novedad y el aprendizaje — biodiversidad y diversidad de instituciones-.

No obstante, la resiliencia es un objeto escurridizo. Para medirla, aunque fuese metafóricamente, es importante especificar la escala temporal y espacial: lo resiliente hoy puede no serlo mañana. Igualmente es crucial hacer evidente la interconexión entre niveles espaciales distintos (Carpenter et alt, 2001). Si bien puede quedarnos relativamente claro el concepto, sobre todo en sus versiones más metafóricas, la operativización no es tarea sencilla. Sin embargo, la resiliencia nos parece una buena heurística para pensar el medio ambiente y a los humanos en él, desde una consideración simultánea del cambio-permanencia en los sistemas adaptativos complejos.

Un sistema socio-ecológico es quizá el epitome de la complejidad. Y la comprensión de su dinámica y funcionamiento es el gran desafío de una ciencia, que no se resigne a la comodidad estéril que le brindan la segmentación, la compartimentación disciplinaria y el pensamiento mecanicista. Sin duda, el éxito de las intervenciones ambientales dependerá de esa comprensión.

\section{Operativizar el estudio de la resiliencia socio-ecológica}

A pesar de que la noción de resiliencia socio-ecológica tiene un alto potencial heurístico, no es fácil hacer un uso operativo de ella en el análisis de contextos específicos: es necesario profundizar en su empleo empírico y contrastar con referencias etnográficas su potencialidad explicativa. Por eso una de nuestras líneas prioritarias de trabajo está siendo la aplicación en estudios etnográficos de algunas de las propuestas teórico-analíticas ya disponibles. Una vez que se ha determinado que un socioecosistema tiene una configuración positiva y que, por tanto, nos preocupa su sostenibilidad, interesaría saber si se encuentra suficientemente capacitado para "seguir siendo sustancialmente como es". Para dilucidar todo ello, no cabe más que comprobar su resiliencia. Este tipo de investigación nos permite también avanzar 
en la aportación teórica que desde la antropología pudiera hacerse a esta línea de investigación transdisciplinar.

En el reciente, pero fructífero, campo internacional de la investigación sobre resiliencia, ya disponemos de algunas propuestas sobre la identificación de los elementos socio-culturales que generan y fortalecen la resiliencia de un socioecosistema (Berkes y Seixas, 2005; Folke, 2003):

1. Aprender a vivir con el cambio y la incertidumbre: capacidad para aprender de las crisis, para construir respuestas a los cambios ambientales, para controlar perturbaciones y desarrollar estrategias que hagan frente a procesos de cambio. A este respecto las instituciones socio-culturales cumplen un papel central, ya que responden y se reforman en virtud de estas crisis, desempeñando un papel protagonista en su manejo.

2. Alimentar la diversidad para la reorganización y la renovación. El mantenimiento de la memoria socio-ecológica, como experiencia acumulada para hacer frente al cambio, desempeña un papel muy importante y proporciona el marco para la capacidad creativa y adaptable. Además fomenta la diversidad de las instituciones para responder a los cambios, creando un espacio político para la experimentación y la confianza entre las partes interesadas y los agentes implicados.

3. Combinar diferentes tipos de conocimiento, incorporar el conocimiento local en la gestión y la toma de decisiones. El conocimiento local y la experiencia sobre la dinámica de los ecosistemas complejos son irremplazables; de ahí la necesidad de involucrar el conocimiento local en combinación con el conocimiento científico en la gestión ambiental.

4. Crear oportunidades para la autoorganización: capital social, redes de comunicación y confianza, a través de la promoción de estrategias participativas capaces de generar conocimiento amplio e integrado, así como mecanismos para el manejo de conflictos. La autoorganización es básica para aumentar la equidad en el acceso y la asignación de recursos, responder a las tendencias externas y sintonizar diferentes niveles de gobernanza.

Como podemos comprobar, los factores/indicadores de resiliencia referidos son muy próximos a algunos de los temas que han sido y están siendo estudiados habitualmente por los antropólogos - particularmente en referencia al conocimiento local o, en un sentido más amplio, a la cultura local—. No lo son menos las referencias a los procesos participativos, o los asuntos relacionados con el funcionamiento institucional, la confianza o la cohesión social. En este sentido, la antropología puede contribuir consistentemente a consolidar esta propuesta analítica y hacerla operativa, sobre todo habida cuenta del déficit del que adolece en términos de definición y concreción, precisamente en los factores de corte sociocultural.

El propio estudio etnográfico, mediante el cual puede accederse al funcionamiento de los socioecosistemas desde dentro y, por tanto, determinar de manera empírica sus dinámicas más íntimas, presenta un gran potencial para alumbrar la relevancia de las propuestas que apuntamos. Pero, junto a ello, no podemos dejar de mencionar aquí lo fructífero que resultaría la integración del 
debate naturaleza-cultura —uno de los clásicos antropológicos-a la construcción transdisciplinar por la que abogan las nociones de resiliencia y socioecosistema. Igualmente supone una contribución relevante a todo este enfoque teórico el desarrollo de la dimensión afectiva e identitaria, que parece estar implícita en la idea de construir confianza colectiva. Sin ella es difícil hacer operativo ese "alimento de la diversidad" o "la creación de oportunidades para la autoorganización", que nos apuntan Berkes y Seixas (2005). El sentido de pertenencia colectiva y los modelos de identificación de las personas que forman parte de un socio-ecosistema, como formas de apego y apropiación, son - junto a otros aspectos que tienen que ver con los componentes biofísicos - elementos clave para comprender el nivel de resiliencia socioecológica del mismo (Davidson-Hunt y Berkes, 2003; Escalera, 2008). Este ámbito de las identificaciones colectivas es una de las áreas en la que los antropólogos han vertido sus esfuerzos y producido importantes contribuciones teóricas, con luces y sombras. Todo ello puede ahora integrarse con un sentido práctico/aplicado en el análisis de la resiliencia, con un alto poder explicativo sobre el desempeño social en situaciones de crisis, sobre la capacidad de autoorganización e incluso en torno al vigor de las formas locales de conocimiento.

En este artículo vamos a mostrar muy sumariamente dos aproximaciones antropológicas a la resiliencia socio-ecológica a través de estudios de caso. En uno de ellos - Agua Blanca - exploramos si el desarrollo del turismo comunitario potencia o no la resiliencia socio-ecológica dentro de un parque natural. Para ello se realiza una investigación etnográfica, que nos ayuda a dilucidar el estado de los factores que propician la resiliencia en una comunidad y su territorio al interior de ese parque. En el segundo caso - Coopesilencio - analizamos cómo la resiliencia corre paralela a un proceso de comunitarización de la sociedad local. Esa "construcción de la comunidad" tiene en su vertiente organizativa las claves para comprender elementos capitales de la resiliencia socio-ecológica: confianza, redes, cohesión, autoorganización... Sólo el desarrollo y mantenimiento de una estructura comunitaria puede explicar la superación de distintos desastres naturales y colapsos económicos, así como la posibilidad de restauración ambiental en un territorio devastado, todo ello a través de respuestas culturales creativas e inesperadas.

\section{6. ¿Puede el turismo ser una estrategia resiliente? Reflexiones desde Agua Blanca $^{4}$}

Agua Blanca es un socio-ecosistema que incluye 260 habitantes que ocupan unas 8000 has. al interior del Parque Nacional Machalilla (Manabí, Ecuador). Este territorio es de gran relevancia ambiental y extremadamente sensible a la acción humana, por eso las actividades antrópicas han sido profundamente vigiladas y transformadas en los últimos decenios. Asimismo, el territorio comunitario es muy rico en restos y ruinas arqueológicas de la cultura manteña, parte de las cuales se

${ }^{4}$ Este epígrafe se basa y puede ser ampliado en Ruiz 2007, 2009 y 2011; así como en Ruiz y Hernández, 2010. El trabajo de campo en Agua Blanca tuvo lugar en distintas campañas entre 2006 y 2008 con financiación de la AECID. 
encuentran excavadas. Agua Blanca oferta actividades turísticas aprovechando estos recursos desde mediados de los pasados años ochenta, aunque su mayor desarrollo se ha alcanzado a partir del 2000 (Ruiz, 2007, 2009). El turismo natural y cultural es la clave, como estrategia de sostenibilidad, de la permanencia de los aguablanquenses en su territorio. Cuando se creó el parque, en 1979, las autoridades intentaron deportar a la comunidad para conseguir un espacio protegido sin presencia humana. Tras un periodo muy conflictivo, se transigió con la presencia de los aguablanquenses en el recinto protegido a cambio de una transformación sustancial de su forma de vida: muy especialmente de la caza, ganadería, tala de madera y sobre todo producción de carbón vegetal.

En Agua Blanca, el turismo se organiza de forma comunitaria. La comunidad tiene la posesión de su territorio y autorregula — con el beneplácito del parque - sus actividades dentro del mismo, incluyendo la actividad turística. No hay competencia externa posible, ya que el territorio comunitario está cerrado a la iniciativa privada. La oferta turística comunitaria es un complemento a la oferta turística del parque en general, centrada en Puerto López, capital del cantón. Aproximadamente un tercio de los visitantes al parque - 33.318 en 2007- visitan también Agua Blanca (Ruiz, 2009: 154). Desde el parque se entiende que el desarrollo turístico de la comunidad tiene como contrapartida la conciencia ambiental de sus habitantes y asimismo implica una vigilancia colectiva sobre un territorio que el propio parque no podría cubrir con efectividad.

Así, los recursos que se usan turísticamente —el medio ambiente y las ruinas arqueológicas - son posesiones de la comunidad y como tales rigen una organización colectiva para atender a los visitantes que llegan a realizar una visita de entre dos y cuatro horas. Esta visita es una de las fuentes principales de ingresos monetarios de los aguablanquenses - $24,5 \%$ del total en 2006-. La organización turística comunal permite un reparto muy plural de los beneficios, que llegan con diferente incidencia a casi el $70 \%$ de las familias. El turismo significa fundamentalmente trabajo como guías; el número de éstos - uno por grupo doméstico para favorecer la mayor participación y reparto de beneficios posible - viene marcado por un umbral de ingresos mínimos que haga a la actividad "rentable". Los ingresos se reparten de forma igualitaria y equitativa entre los guías. El trabajo turístico se organiza en turnos de diez días, en los que los guías no pueden trabajar en ninguna otra actividad. Por tanto, el beneficio obtenido debe justificar esta exclusividad en la ocupación, al tiempo que autorregula el número de aguablanquenses que trabajan como guías -30 en 2008-.

En la comunidad se desarrollan otras actividades - agricultura para autoconsumo, construcción, guías del parque, carboneo, recolección de frutos silvestres, ganadería...-, que de forma complementaria permiten un umbral de subsistencia que los habitantes consideran razonable, al punto de desistir de las tradicionales estrategias migratorias. El turismo tiene un papel fundamental en la viabilidad de este sistema económico.

Por otra parte, y frente a las seculares tensiones entre la comunidad y el parque por la conservación del medio, el desarrollo del turismo ha ido acompañado de un 
cambio sustancial en las formas de relación de la comunidad con el entorno. Hasta los pasados años 70, el territorio comunitario formaba parte de una hacienda agroganadera que lo explotaba con una orientación exclusiva de mercado. Cuando la hacienda desaparece, sus trabajadores, los miembros de la comunidad, permanecieron en el lugar con pautas de explotación similares, aunque centradas en las actividades más negativas para el medio — caza, extracción de madera y carboneo- Ello causó un fuerte impacto ambiental creciente. Sin embargo, desde inicios de siglo, la propia dirección del parque constata una recuperación notable tanto en la cubierta arbórea del valle del río Buenavista como en la presencia de aves y pequeños mamíferos. Sin abandonar completamente algunas de las prácticas más lesivas para el medio - carboneo y ganadería - , su reequilibrio con la actividad turística permite una complementariedad de actividades que ha rebajado sensiblemente la presión sobre el entorno, permitiendo su regeneración.

Resulta evidente que la actividad turística ha supuesto un cambio en el devenir de este territorio, y que podemos aventurar que el socioecosistema resulta más sostenible que hace apenas diez años. Pero, ¿el turismo tiene relación con la resiliencia del socio-ecosistema? ¿En qué sentido? Para responder a estas preguntas, no entramos a valorar el propio entorno, sino más bien cómo se funciona en él: el marco de las relaciones socio-ambientales. Al estudiar la resiliencia, no tratamos de retratar una situación ambiental, a modo de foto fija del territorio, sino de elucidar la manera colectiva de proceder sobre el entorno. Ese es el secreto de la sostenibilidad: constituye una forma más que un contenido. Si el socio-ecosistema es resiliente, garantizaremos la sostenibilidad, ya que será capaz de mantener sus elementos sociales y ambientales básicos en el futuro, a pesar de los sobresaltos que pudieran darse. Para ello hemos de tener bien presente: (1) el protagonismo humano en el devenir del socioecosistema; y (2) que la resiliencia es una característica del conjunto del socio-ecosistema, no de sus partes. Analizado desde esta perspectiva, el turismo comunitario en Agua Blanca sería una actividad que facilitaría o no la resiliencia del socio-ecosistema - y por ende su sostenibilidad - en la medida en que contribuyera a las pautas que nos proponen Berkes y Seixas (2005) y Folke (2003) como sustentadoras de la resiliencia. Éstas han sido expuestas unas páginas más arriba, ahora procedemos a recorrer someramente la comunidad y su actividad turística desde esa lógica.

¿Aprender a vivir con el cambio y la incertidumbre? En Agua Blanca, turismo comunitario significa fundamentalmente trabajar como guía. Esta actividad está marcada por un umbral de rentabilidad individual sujeto constantemente a los cambios en la demanda, y asimismo a la incertidumbre por cuanto los ingresos experimentan notables variaciones estacionales. La actividad turística en sí no crea estabilidad, sino todo lo contrario: permanente fluctuación de rentabilidades, estrategias y tácticas individuales y domésticas. Pero el turismo comunitario en Agua Blanca tiene una vocación de complementariedad. No hay familia ni individuo que centre sus ingresos exclusivamente en el turismo, no porque no fuera posible hacerlo, sino porque esa complementariedad es una estrategia colectiva que tiene como objetivo, precisamente, lidiar con el cambio y la incertidumbre para la permanencia en el 
territorio. Por eso se busca que beneficie al mayor número de grupos domésticos y se reparte el trabajo entre el mayor número de gente considerando umbrales mínimos de rentabilidad.

En la comunidad hemos catalogado un total de 22 actividades productivas diferentes. Los grupos domésticos que no participan del turismo comunitario desarrollan una media de 4,6 actividades distintas a lo largo del año; sin embargo, los grupos que participan del turismo comunitario implementan una media de 6,8 actividades en el mismo periodo; es decir, un 50\% más de actividades productivas diferentes. Los primeros son más dependientes de la extracción directa de recursos del medio, los segundos combinan esa extracción con las actividades turísticas, lo que ya de por sí les obliga a "extraer" de forma más extensiva. Por tanto, la actividad turística anima la complementariedad y diversidad de actividades, y asimismo reduce la dependencia extractiva. Esta estrategia facilitaría la reacción ante posibles crisis en algunos de los recursos disponibles, ya que se sostiene en un rápido feedback de respuesta a situaciones cambiantes, construido sobre un manejo permanente de las variaciones. El hecho de que en los últimos años el catálogo de las actividades económicas, en vez de reducirse, se haya ampliado facilita la capacidad para lidiar con el cambio y la incertidumbre, y en ello juega un papel fundamental la actividad turística comunitaria.

¿Nutrir la diversidad para la reorganización y la renovación? Memoria y confianza como prácticas de la comunidad. La experiencia turística en Agua Blanca consiste en mostrar las claves ecológicas del medio y los rasgos de la cultura manteña, a la que pertenece la comunidad. La memoria colectiva constituye así un elemento fundamental y el desarrollo del turismo ha supuesto una recuperación general de la memoria, que no sólo se transmite a los turistas, sino que previamente se les enseña a los propios guías. A lo largo de los últimos veinte años, han ejercido como guías unos cincuenta aguablanquenses, que han manejado con soltura el conocimiento local sobre la cultura y el medio — los tiempos de la hacienda, las migraciones, las sequías y los fenómenos del Niño- . De esta manera se garantiza la alimentación de la memoria socioecológica como fuente de innovación y novedad.

De la misma manera se potencian instituciones para responder al cambio y se generan espacios para la experimentación (Berkes y Seixas, 2005). El turismo en Agua Blanca está organizado en torno a un comité dependiente de la asamblea de la comunidad. Otras actividades productivas se organizan también de forma similar. Los recursos son colectivos y, si un grupo de miembros de la comunidad se los apropia de manera particular, la asamblea le obliga a constituir un comité que regule su uso. De esta forma se conjuga organización y control. Esos comités son espacios de reflexión, experimentación y construcción de confianza. Concretamente, el comité que organiza el funcionamiento turístico en Agua Blanca sirve de modelo a la comunidad, y sus reuniones son un espacio destacado de la política local. Los grandes asuntos locales de los últimos años tienen alguna relación con el turismo y, en el seno del comité que lo organiza, hemos podido constatar una comunicación directa y fluida que anima a la toma de decisiones y a la acción colectiva. Con todo ello la actividad turística consolida, por un lado, la memoria como práctica y, por otro, la 
confianza mutua, ambas alimentan la diversidad para la reorganización y renovación potenciales, claves en la resiliencia del socio-ecosistema.

¿Combinación de diferentes formas de conocimiento? el liderazgo de los guías turísticos. Los guías ocupan una posición estratégica en la vida comunitaria a diferentes niveles, y en especial en la combinación de distintas formas de conocimiento - como lo hacen en la complementariedad de actividades - un factor crucial - según los teóricos - para el fortalecimiento de la resiliencia socio-ecológica y, por ende, de la sostenibilidad.

A lo largo de los últimos veinte años, los guías han adquirido prestigio no sólo por el acceso a ingresos monetarios importantes - las familias en mejores condiciones económicas son invariablemente las que participan en el turismo-, sino por el propio carácter, sentido e implicaciones de esta actividad. Los guías conforman una suerte de bisagra entre la comunidad y el exterior, no sólo porque sirven de representantes de ésta ante los turistas, sino porque asimismo su condición de guías les obliga a entender los lenguajes usados a uno y otro lado de los límites de la comunidad, y además deben manejar distintos tipos de conocimiento. Los guías están en constante aprendizaje a todos los niveles: son conscientes de lo que los turistas quieren, de los objetivos de la comunidad, de las potencialidades y limitaciones del medio..., de alguna manera encarnan lo que el turismo significa localmente y se convierten en una suerte de "agentes de resiliencia". Los guías son la piedra angular del turismo comunitario en Agua Blanca y, por tanto, protagonistas del efecto resiliente que éste pudiera tener en el sistema socio-ecológico. Si la resiliencia —como hemos visto - se materializa en actitudes y prácticas, los guías - la mayoría de ellos cabezas de familia - encarnan en sus cotidianidades esas actitudes y prácticas resilientes. Son los más conscientes desde el punto de vista ambiental y se convierten en los transmisores de esa conciencia al conjunto de la comunidad, contribuyen destacadamente a construir capacidad para monitorear el medio y gestionarlo participativamente, generar instituciones que enmarquen el aprendizaje, la memoria y la creatividad, crear mecanismos para compartir conocimiento a distintas escalas, y combinar el conocimiento científico y el local (Berkes y Seixas, 2005).

¿Crear oportunidades para la autoorganización? En Agua Blanca el turismo es producto de la autoorganización. Teniendo en cuenta las presiones foráneas - en el pasado y en la actualidad - así como el asesoramiento externo en algunos momentos, lo cierto es que el turismo comunitario en esta comunidad es hoy por hoy la emergencia de un proceso interno de autoorganización. Para la resiliencia la capacidad de autoorganización es un principio básico y el comité que organiza el turismo es un claro ejemplo de esa autoorganización. Con disciplina, eficiencia y eficacia que sorprenderían en la propia empresa privada, el comité organiza la actividad turística en Agua Blanca. Un riguroso reglamento, lleno de prohibiciones y exigencias, y una práctica de debates y sanciones encaradas colectivamente (Ruiz, 2009: 363-378) hacen al comité capaz de articular el trabajo de treinta personas y gestionar sus ingresos mensuales con un equipo directivo, que se renueva cada año y por el que, con el tiempo, han pasado la mayoría de los guías. En este sentido, el comité es una institución que enseña cómo construir acción colectiva en todas sus 
fases: reflexión, debate, planificación, ejecución y evaluación. Además resulta ser un importante contexto de generación de recursos monetarios. No es, por tanto, difícil comprender su centralidad. El comité sigue una estrategia rigurosamente participativa y basada en el consenso para gestionar los recursos colectivos; lo cual no excluye el conflicto y los intereses individuales, sino que más bien asegura un marco para gestionarlos de una manera ágil y, en la mayoría de los casos, efectiva. Las reuniones diarias de los diferentes grupos de trabajo, así como la reunión mensual para repartir los ingresos entre todos los guías, sirven como contexto en el que se socializan todos los problemas y quejas.

Habida cuenta de la importancia del turismo para la vida en Agua Blanca, el comité impone un estilo al funcionamiento colectivo basado en la autoorganización. Esa autoorganización tiene un principio fundamental: la equidad en el acceso y reparto de los recursos. Hemos señalado anteriormente que, para el caso del turismo, esa equidad se entiende en función de un umbral de rentabilidad mínimo. Gran parte de los debates y decisiones colectivas vienen precisamente de la determinación del mismo, lo cual implica automáticamente la ampliación o reducción del número de personas que pueden participar de la actividad.

Como hemos mostrado de forma muy sucinta, el turismo comunitario en Agua Blanca es, más que una mera actividad económica, un marco actitudinal y práctico en la relación entre los propios miembros de la comunidad y de éstos con el medio. En definitiva, contribuye a desarrollar una manera específica de encarar el futuro. Aquí anida la resiliencia. La actividad turística en esta comunidad propicia una forma de estar, de enfrentarse a la vida y a la subsistencia en un entorno frágil. Ésta es la base de la resiliencia: una forma, una actitud, no tanto un estado. A la vista del análisis, podemos afirmar que para Agua Blanca, como socio-ecosistema, la actividad turística - peculiarmente concebida y practicada en forma de turismo comunitario- anima su resiliencia, ya que contribuye a que los miembros de la comunidad vivan con el cambio y la incertidumbre en mente, alimenten la diversidad del socio-ecosistema, combinen diferentes tipos de conocimiento y creen oportunidades de autoorganización sobre la base de la equidad en el acceso y reparto de los recursos. Desde aquí se moldea una específica actitud humana respecto al entorno que puede hacer al conjunto "sostenible".

El turismo en Agua Blanca alimenta la sostenibilidad del socio-ecosistema en la medida en que incrementa su capacidad resiliente. En la actualidad, la actividad turística ha contribuido a reducir otras actividades que causaban un impacto notable sobre el medio y ponían en peligro la propia pervivencia de la población. Por ejemplo, a principios de los pasados años 80 , para la práctica totalidad de las familias, la principal fuente de ingresos residía en el carboneo o en la tala de madera; en 2006 sólo seis familias — de las 59 que forman la comunidad— tenían en el carbón su fuente de ingreso principal y ninguna comerciaba con la madera. Asimismo la presencia de ganado caprino en el territorio de la comunidad se ha reducido en casi el 70\% entre 1997 y 2007 (Ruiz, 2009: 192), y la caza es una actividad puramente testimonial. Esta evolución hubiera sido imposible sin el desarrollo turístico comunitario, que ha abierto también un camino sólido para ampliar la dependencia sobre 
recursos múltiples, aumentando, al mismo tiempo, el nivel de vida de los habitantes de la comunidad.

Para las autoridades del parque nacional, la conservación del medio en el territorio de la comunidad sigue un curso razonable. Para la propia comunidad, el nivel de vida y consumo del que disfrutan es aceptable al punto de que es opinión prácticamente unánime de hombres, mujeres y jóvenes que la comunidad es un espacio más atractivo para vivir que la gran ciudad, y esto viene de personas que mayoritariamente han vivido la emigración. Por tanto, partimos de un sistema que tanto para las autoridades ambientales como para sus habitantes merecería la pena conservar; pero ¿hasta qué punto es sostenible?

En la actualidad, la vida en esta comunidad está marcada fundamentalmente por su modelo de turismo comunitario. ¿Qué sucedería si desaparece la actividad turística? ¿O si aumenta la demanda de visitas de forma inabarcable? ¿O si sobreviene una sequía prolongada? Cualquiera de estas circunstancias conllevaría una transformación drástica en las actuales condiciones de vida. Pero cabe pensar que no se transformarían aspectos fundamentales para la sostenibilidad del socio-ecosistema como la complementariedad de actividades económicas, la equidad en el acceso comunitario a los recursos - fueran estos los que fuesen-, o la consciencia de los límites ambientales. Sobre estos factores descansa la sostenibilidad del medio, entendida de forma co-evolutiva.

El análisis nos muestra que las instituciones colectivas, las formas de organización comunitarias, las actitudes y prácticas individuales, que el turismo comunitario moldea, servirían para encarar las variaciones imprevistas que afectaran al socioecosistema, de manera que el socio-ecosistema mantuviera su forma esencial, que toma cuerpo en el modo en que sus habitantes humanos lo habitan y conciben. Por tanto, no es el turismo per se lo que alimenta la resiliencia, sino su forma organizativa, en este caso su carácter comunitario. Este carácter podría extenderse a cualquier otra actividad que en el futuro fuera estratégica para el funcionamiento colectivo. Ahí reside la clave de la resiliencia y la sostenibilidad de este socioecosistema.

\section{Coopesilencio: comunitarización y resiliencia ${ }^{5}$}

Coopesiliencio es un buen ejemplo de colectivo humano que, resiliente con respecto a las crisis económicas, sociales y políticas — derivadas de las condiciones históricas estructurales - y medioambientales — consecuencia de las características ecológicas de la región en que se enclava-, actúa como un factor decisivo en la resiliencia global del socioecosistema del que forma parte, contribuyendo a su preservación e incluso restauración.

${ }^{5}$ El trabajo de campo en Coopesilencio se ha llevado a cabo en el marco de los proyectos "Turismo, recreaciones ambientales y sostenibilidad en los espacios protegidos de Andalucía: resiliencia socioecológica, participación social e identificaciones colectivas" (P06 RNM 02139), financiado por la Consejería de Innovación, Ciencia y Empresa de la Junta de Andalucía y "Turismo sostenible, desarrollo local y cooperación internacional: resiliencia socioecológica y articulación regional transfronteriza en el Río San Juan (Costa Rica-Nicaragua)" (A/5445/06), financiado por la AECID. Para ampliar información, ver Escalera 2008, 2011. 
La cooperativa de campesinos Coopesilencio está en El Silencio, una localidad ubicada en el curso bajo del río Savegre, en la vertiente del Pacífico central costarricense, a unos de $30 \mathrm{Km}$. al sur de Quepos y del Parque Nacional Manuel Antonio. El Silencio agrupa a unas 80 familias con una población total de casi 500 habitantes. La mitad de ellas - 42 familias - están asociadas en Coopesilencio, una organización definida por los principios de la autogestión, que además es depositaria de la titularidad de la propiedad de la tierra y de todos los medios de producción, considerados colectivos e indivisibles. Coopesilencio es una estructura comunitaria dentro de la localidad de El Silencio. Las 42 familias socias conforman una comunidad no sólo desde el punto de vista simbólico, sino principalmente organizativo, que tiene a la asamblea de socios como órgano soberano en la toma de decisiones en lo relativo a la producción, pero también en relación a otras esferas de la vida colectiva. Desde nuestra perspectiva analítica, esta cooperativa insufla una particular forma de funcionar al conjunto de la localidad de El Silencio, lo que anima un proceso de “comunitarización” local. Evidentemente, no seguimos aquí una visión de comunidad como ente estático y esencial, sino que de una forma operativa, entendemos lo comunitario más bien como un proceso, como el despliegue de un conjunto de mecanismos particulares para el funcionamiento social (Brint, 2001; Ruiz, 2009; Walsh y High, 1999). Nuestra investigación —en curso - en torno a Coopesiliencio y El Silencio considera la comunitarización de las relaciones sociales como un factor primordial para comprender el devenir del socioecosistema, en el que se inserta y al que pertenece; por tanto, como un indicador de resiliencia socio-ecológica.

El Silencio se ubica en una región de clima tropical húmedo, en la que la vegetación originaria predominante era el bosque tropical seco, en la costa, y el bosque lluvioso, conforme se asciende hacia el interior. De ambos quedan todavía zonas sin transformación importante. Desde el punto de vista del funcionamiento ecológico del sociecosistema, deben señalarse dos factores que tienen una incidencia capital y que marcan su carácter "catastrófico": el paso periódico de huracanes caribeños y ciclones del Pacífico, y los desbordamientos del río Savegre. Estos fenómenos determinan el alto grado de incertidumbre que presenta el medio para la vida de los animales, vegetales y humanos que constituyen el socioecosistema de la región.

Precisamente en 1955, como consecuencia de un desbordamiento especialmente severo del río, se vio asolada buena parte de las plantaciones de banano, con las que la United Fruit Co. había sustituido desde principio del siglo XX importantes extensiones del bosque primario primigenio. El resultado de la destrucción fue el abandono del cultivo y el arrendamiento de las zonas más altas a algunos particulares para su aprovechamiento como pastos. No obstante, parte de los antiguos trabajadores y colonos de la compañía permanecieron en el entorno subsistiendo de manera precaria. A principios de los pasados años 60 , los trabajadores y pequeños campesinos, que resistieron la debacle bananera, iniciarán un proceso que, partiendo de la idea de comprar la tierra a la compañía propietaria, y ante la negativa de ésta a venderla, derivó a principios de los últimos años 70 en una movilización, que tendrá como punto culminante la ocupación que 42 familias de campesinos hicieron de una finca denominada El Silencio. Se sucedieron ocupaciones y expulsiones: se entraba 
de noche en la finca, se construían improvisados ranchos de ramas que terminaban siendo quemados por los empleados de la compañía y la policía costarricense; consecuentemente sus ocupantes eran desalojados. En 1973, el gobierno costarricense, a través del Instituto de Tierras y Colonización (ITCO), negociará con la compañía la compra de la finca para cederla a continuación a los campesinos, constituidos, como condición para ello, en cooperativa agropecuaria, Coopesilencio (CEPAL, 1999; Cordero, 2006; Gertler, 2001; Marín, 1999; Sobrado, 1998). Los testimonios de algunas de las personas que intervinieron en las ocupaciones ponen de manifiesto la intensidad de aquellos momentos y la impronta que han dejado en ellas en particular y, por extensión, en el conjunto de la población en general. Este periodo de lucha por la tierra contribuirá decisivamente a la creación de una fuerte vinculación con el lugar y a la generación de un sentimiento de comunidad entre los integrantes del grupo de familias participantes.

A la constitución de la cooperativa, que contó con el apoyo del Instituto de Desarrollo Agrario costarricense (IDA), siguió un difícil periodo para sus socios. Debían participar de un tipo de organización para el que ninguno de sus miembros estaba preparado: habían sido durante toda su vida jornaleros agrícolas. En ello fue fundamental el acompañamiento que recibieron por la Universidad Nacional, a la que el gobierno encargó el asesoramiento y la capacitación de los cooperativistas.

Desde 1985 la principal actividad de Coopesilencio es el cultivo de palma africana para la producción de aceite, que ocupa actualmente 530 ha. ${ }^{6}$ de las 1000 ha. que constituyen el terreno propiedad de la cooperativa. En esta finca se han dedicado 300 ha. a bosque primario y el resto a terreno de pastos, para alimentar al ganado vacuno de la cooperativa - 80 cabezas- También existe una huerta agrobiológica y otros aprovechamientos agroganaderos menores, la mayor parte de ellos para autoconsumo: porqueriza, granja avícola...

En distintos momentos se han ensayado otras producciones agrícolas - arroz, soja, tabaco, papaya, cacao... - que no prosperaron, sea por las condiciones medioambientales, por los elevados costes de los insumos agroquímicos que algunas de ellas requerían o por las dificultades de comercialización. Como consecuencia, la cooperativa tiene su eje principal de actividad en el fruto de la palma — coyolt—. Su producción se cifra en torno a las 2.000 toneladas al año, que venden a la empresa Palmatico, cuya planta de transformación se encuentra en la zona. Esta producción supone entre el 75 y el $80 \%$ de los ingresos de la cooperativa. La palma es un cultivo con un impacto ambiental escaso en comparación a la mayoría de los cultivos industriales que se dan en el país — banano, piña...-, y que genera mucha demanda de mano de obra, siendo además un cultivo que resiste bastante bien a plagas ${ }^{7}$ y

${ }^{6}$ La superficie plantada y en producción varía, ya que el proceso vegetativo de las palmas requiere, para dar producción, cuatro años desde su plantación. Las plantas alcanza su mayor nivel entre los 6 y los 15 años y decaen drásticamente a partir de los 20-25, por lo que son eliminadas a esa edad. Se reinicia entonces el proceso de plantación.

${ }^{7}$ No obstante, en la actualidad, la producción se ha visto perjudicada notablemente por la enfermedad de la flecha seca que ha afectado a las plantas. Ello constituye un nuevo factor que empuja a la cooperativa a la diversificación de actividades. 
condiciones meteorológicas adversas. El trabajo de cuidado de las plantaciones, recogida del fruto y transporte a la planta de transformación es realizado regularmente por un grupo de unos 35 hombres, 20 de ellos asalariados y el resto socios. No obstante, en los meses de mayor producción, la participación de mano de obra temporal es relativamente importante, participando incluso mujeres y niños de la localidad.

Coopesilencio R.L. opera bajo el modelo de autogestión, que lo diferencia de las cooperativas tradicionales en cuatro principios básicos:

1. Propiedad comunal: la titularidad de la propiedad de los medios de producción, tierras, proyectos productivos y activos en general es colectiva e indivisible.

2. Trabajo compartido: el aporte de los asociados es la mano de obra en los diferentes proyectos sociales productivos o ambientales. El trabajo se distribuye entre los socios en función de las necesidades, los deseos y la cualificación.

3. Participación democrática: todos los miembros de la cooperativa poseen los mismos derechos y las mismas obligaciones, y tienen voz y voto en la toma de decisiones.

4. Participación económica: los costes y beneficios son compartidos entre todos los miembros de manera igualitaria. En principio, el salario era también único, pero progresivamente y fruto de las distintas funciones laborales, desarrolladas conforme se fueron diversificando las actividades de la cooperativa, se estableció una proporción de 1 a 3 en función de las características y responsabilidades de las tareas llevadas a cabo.

La cooperativa es presidida por un Consejo formado por cinco miembros que se renuevan cada dos años, con una sola posibilidad de reelección. La gestión, estrictamente empresarial, es confiada a un gerente contratado, mientras que el gobierno de los asuntos comunitarios se lleva a cabo por distintos comités integrados por miembros de la cooperativa: seguridad, educación, comisariato - almacén cooperativo-, etc... Para el desarrollo de sus actividades, cabe la posibilidad de la contratación de trabajadores por cuenta ajena, fundamentalmente de El Silencio.

En 1998 la cooperativa vivió una tesitura crítica. Algunos de sus miembros fundadores pretendían disolverla, dividiendo la propiedad. Esta circunstancia fue habitual en algunas de las experiencias de cooperativismo agrario establecidas en Costa Rica desde los pasados años sesenta. Entonces, otra catástrofe, esta vez provocada por el huracán Mitch ${ }^{8}$, destruyó gran parte de las plantaciones de palma. La tragedia tuvo un efecto paradójico. Por un lado, acentuó la crisis de la cooperativa con la interrupción del suministro de agua potable y electricidad y las dificultades de comunicación debido a la destrucción del puente sobre el río Guabo, que supuso durante mucho tiempo un grave obstáculo para dar salida a la producción. Pero, por otra parte, impulsó el desarrollo de un proyecto de agro-ecoturismo comunitario

\footnotetext{
${ }^{8}$ Antes, en 1986, el huracán Juana y el César, en 1995, causaron también importantes daños en las plantaciones.
} 
que, con el tiempo, se ha convertido en un revitalizador de la cooperativa y pilar de su incidencia socio-ambiental.

Ante el desastre y en vez de renunciar al proyecto de desarrollo colectivo, la junta directiva del momento, apoyada por la mayoría de la asamblea, decidió aprovechar la madera procedente del arrasamiento, que el huracán había supuesto en una zona importante del bosque para, con el trabajo voluntario de sus miembros, construir las primeras cabañas, núcleo originario del actual albergue. Se levantaron así los primeros edificios del complejo turístico que gestiona la cooperativa. Incluso el mobiliario se construyó con la madera de los árboles arrasados. Todos estos trabajos fueron realizados por artesanos de la propia localidad. La diversificación de actividades que supuso el agro-ecoturismo comunitario ha permitido frenar la salida de más miembros de la cooperativa y la localidad, e incluso revitalizarla, dando oportunidad de trabajo a un grupo creciente de mujeres y también a algunos jóvenes que encuentran en la actividad turística mayor atractivo que en el trabajo agrícola. Este factor de retención demográfica en El Silencio frena un éxodo juvenil que, de otro modo, sería prácticamente total.

En 2005 se produjo un nuevo huracán, en este caso el Rita, que destruyó una parte importante del palmeral, mermando de manera significativa la producción del principal recurso económico, y destruyendo también lo que para cualquier comunidad costarricense representa el centro de la vida social: la plaza-campo de fútbol. Ante ello, los socios reaccionarán nuevamente potenciando el proyecto turístico, renegociando las deudas acumuladas y trabajando colectivamente para recuperar su plaza-campo de fútbol, sentida y añorada como símbolo de la identidad comunitaria.

La catástrofe no sólo tuvo un fuerte impacto en la producción agrícola, sino que los daños causados en la pequeña infraestructura, construida para atender la oferta agro-eco-turística, estuvieron a punto de hacer desistir a la cooperativa de continuar con esta actividad, cediendo su explotación a algún operador externo. El empeño de algunos de los líderes de la cooperativa en no renunciar al control del turismo sirvió, en última instancia, para potenciarlo de nuevo. Se ampliaron las instalaciones con la construcción de algunas cabañas más y un bar-restaurante. Asimismo se apoyo la formación específica de algunos jóvenes y mujeres para una mejor atención a los visitantes, y se puso en marcha una experiencia de alojamiento con algunas familias de la cooperativa. Todo ello evita, al menos por el momento, la pérdida del control de la cooperativa sobre la actividad turística. Actualmente Coopesilencio dispone de diez habitaciones con una capacidad para 50 huéspedes.

Paralelamente, la cooperativa ha intentado desarrollar otras líneas de actividad, a fin de diversificar las fuentes de financiación y aumentar así su capacidad de resistencia frente a la fuerte dependencia de la industria de transformación del fruto de la palma para la obtención de aceite. En este sentido, es importante tener en cuenta el desarrollo de distintos proyectos de perfil medioambiental que, amén de suponer una fuente de empleo, restaura el medio ambiente y lo hace más atractivo de cara a la actividad turística. Uno de ellos es el Centro de Rehabilitación para la Vida Silvestre del Río Savegre, que lleva a cabo programas de rescate, rehabilitación y 
reintroducción de animales salvajes 9 . Otro está relacionado con la conservación de zonas de bosque primario y la reforestación con especies como la teca, el eucalipto, la melina, el cedro y el laurel para la producción sostenible de madera ${ }^{10}$. En el marco de este último proyecto, además de su vocación conservacionista, se contempla también el desarrollo de una serrería y la fabricación de muebles para ampliar las posibilidades de empleo. Asimismo, se ha construido el Centro de Educación y Capacitación Ambiental (CECADES) ${ }^{11}$, en cuyas instalaciones se desarrollan actividades formativas, educativas y de investigación.

Este recorrido por el devenir de El Silencio, y más específicamente de Coopesiliencio, nos muestra con nitidez el sentido socio-cultural de factores como la flexibilidad, la innovación, el aprendizaje, la reorganización, la creatividad, la experimentación..., cuyos ecos deben resonar desde la primera parte de este artículo. Coopesiliencio, en su evolución, contribuye plenamente a dos de las principales características de un socioecosistema resiliente: capacidad para vivir con el cambio y la incertidumbre, y la creación de oportunidades para la reorganización. En definitiva, El Siliencio se nos muestra como un socioecosistema con indudable capacidad de "adaptación creativa".

El socioecosistema, del que forma parte Coopesilencio, está sometido a una dinámica marcada tanto por las fluctuaciones del mercado internacional como por distintos desastres naturales. La resiliencia de ese ecosistema implica la capacidad de dar respuesta a ambas dinámicas sin por ello disolverse o desaparecer. La evolución de la zona en los últimos cincuenta años muestra un devenir marcado por una notable capacidad para lidiar con el cambio y la incertidumbre, cuando lo más esperable hubiera sido quizá el abandono de la población, tanto tras la retirada de la compañía bananera como después de cada uno de los huracanes que asolaron la zona. Frente a las adversas coyunturas económicas y ambientales, las respuestas locales tienden hacia un claro proceso acumulativo de diversificación productiva, que paradójicamente es el mejor garante de la sostenibilidad ambiental, pues impide una excesiva especialización en cualquier monocultivo que, sin duda, tendría efectos perjudiciales sobre la biodiversidad ${ }^{12}$. La misma pervivencia de la empresa bananera nos hubiera legado un socioecosistema mucho más pobre desde el punto de vista ambiental: la comarca estaría completamente deforestada como consecuencia de la expansión de la frontera agrícola. Pero es más, dentro de esa diversificación, las

\footnotetext{
${ }^{9}$ Es en especial el caso de la lapa roja — ara macao, especie de guacamayo en peligro de extinción - , con el apoyo de organismos como el SGP (Small Grants Program) del Fondo GEF (Global Environment Facility) e impulsado por el Programa de las Naciones Unidad para el Desarrollo (PNUD).

${ }^{10}$ Cuenta con el apoyo del MINAET (Ministerio Costarricense de Naturaleza, Energía y Telecomunicaciones), en el marco del programa de pago por servicios ambientales, dentro del que es tramitadora oficial del Certificado de Protección de Bosque, incentivo dado en el país a personas físicas o jurídicas interesadas en mantener su bosque sin alteración, dedicado a un uso suave, como es el ecoturismo, y en colaboración con entidades y organizaciones nacionales e internacionales, como la AECID, la cooperación holandesa o la japonesa.

${ }^{11}$ Cuenta con el apoyo de la AECID y la Xunta de Galicia.

${ }^{12}$ Incluso el abandono sin más de un espacio, ya fuertemente antropizado, propiciaría una evolución problemática del mismo: especies oportunistas, suelos alterados...
} 
últimas tendencias y estrategias de Coopesilencio apuntan a la directa regeneración ambiental de las 300 ha. de bosque primario que posee la organización. Tanto las oportunidades de financiación externa como las consecuencias turísticas de estos proyectos apoyan sustancialmente esta línea estratégica de actividad.

El caso de Coopesilencio muestra cómo el empeño de un grupo humano en mantenerse en la tierra, a la que accedieron con su lucha, y su determinación en no renunciar a la organización comunitaria, dividiendo la propiedad común — según ha sucedido en otros casos-, no sólo ha permitido el sustento autónomo de un número importante de familias, sino que, implícitamente, influye en la conservación y regeneración del medio. Coopesilencio aparece como un factor que fortalece la resiliencia del socioecosistema en el que se inserta y ello tiene su base precisamente en el modelo organizativo de la cooperativa.

Coopesiliencio supone para la localidad de El Silencio un vector de comunitarización. Si bien no es toda la localidad la que pertenece a la cooperativa, no es menos cierto que esta institución marca la pauta del funcionamiento colectivo, sea como organización más dinámica de la localidad o como modelo organizativo para el conjunto del pueblo. La cooperativa irradia su estilo comunitario de gestión colectiva hacia el conjunto de la población local. Y en ese estilo adivinamos la creación de oportunidades para la autoorganización. Coopesiliencio nos muestra a lo largo de su existencia que es el marco más fuerte dentro del socioecosistema para generar capital social, redes de comunicación y confianza, a través de la promoción de estrategias participativas capaces de generar conocimiento amplio e integrado, así como mecanismos para el manejo de conflictos. Esa autoorganización es básica para aumentar la equidad en el acceso y la asignación de recursos, responder a las tendencias externas y sintonizar diferentes niveles de gobernanza - desde las organizaciones internacionales, pasando por el Estado hasta llegar al nivel local- . Nuestra investigación apunta que Coopesilencio induce en la localidad de El Silencio un proceso de comunitarización de la vida social, que contribuye al aumento de la resiliencia del socio-ecosistema. La capacidad social para reponerse a desastres naturales, desde la perspectiva de la resiliencia socio-ecológica, ya ha sido estudiada (Adger et alt., 2005) y sus conclusiones se evidencian en el caso de El Silencio. Asimismo, la vinculación de modelos específicos de socialidad humana a formas de sostenibilidad (Honborg, 2001), nos hace pensar que la comunitarización puede ser un marco de relaciones sociales especialmente resiliente desde el punto de vista socio-ecológico. Aunque no es lugar para extendernos en ello, cabe tenerse en cuenta que entendemos lo comunitario como un marco analítico conformado por mecanismos relacionales precisos (Brint, 2001; Ruiz et alt, 2008), que no tiene porqué estar vinculado a sociedades tradicionales, ancestrales o indígenas, tal como nos muestra el ejemplo que estamos tratando. La comunitarización de la vida social es una táctica que permite habitar un entorno y que parece aumentar la resiliencia del conjunto del socioecosistema, con efectos sociales y también biofísicos. 


\section{Resiliencia y Antropología Ambiental}

Es precisamente esta perspectiva la que más se echa en falta en los trabajos sobre resiliencia: el análisis de las condiciones sociales de la sostenibilidad, que es tanto como decir el estudio de las condiciones sociales de la resiliencia socio-ecológica. Ésta es una de nuestras líneas prioritarias de estudio, tal como muestran los casos de Agua Blanca y Coopesiliencio, y sobre lo que seguimos trabajando ahora en algunas de las islas del archipiélago de las Galápagos y en distintas zonas de Andalucía ${ }^{13}$. Para nosotros, los ámbitos locales en los que trabajamos son magníficos laboratorios en los que estudiar a nivel micro los aspectos más relevantes de la configuración bio-fisico-cultural del medio ambiente y, por ende, de las condiciones de su sostenibilidad. Es en ellos dónde podemos encontrar, tras una detallada investigación etnográfica, la evidencia de procesos y tendencias que nos permitan arrojar luz sobre los problemas relacionados con la conservación ambiental y el desarrollo social.

Nos parece que el concepto de resiliencia socio-ecológica y toda la perspectiva teórica que lo acompaña constituyen un marco analítico muy apropiado para estudiar la articulación entre humanos y medio ambiente y, por tanto, para enriquecer la antropología ambiental. La relación entre antropología y teoría de la resiliencia es doble. Si bien ésta última puede sugerirnos nuevas perspectivas más holísticas, capaces de superar operativamente estériles dicotomías naturaleza-cultura, la primera ofrece a la investigación transdisciplinar el valor de la etnografía y del estudio micro para la comprensión más general y profunda de los fenómenos socio-ambientales.

\section{Referencias bibliográficas}

ABEL, Thomas; STEPP, John Richard

2003 "A new ecosystem ecology for Anthropology". Conservation Ecology, 7, 3: 12.

ADGER, W. Neil; HUGHES, Terry; FOLKE, Carl; et alt.

2005 "Social-ecological resilience to coastal disasaters". Science, 309: 1036-1039.

BARRANTES, C.; VICTOR, J.

1998 La Construcción de un Sueño. Coopesilencio, 25 Años Después. Heredia, C.R.: EUNA.

BERGSON, Henri

2008 L'evolution creatrice. Paris: PUF.

BERKES, Fikret; SEIXAS, Cristiana

2005 "Building resilience in Lagoon Social-ecological systems: a local-level perspective". Ecosystems, 8: 967-074.

${ }^{13}$ Lo hacemos en el primer caso dentro de los proyectos: "Antropología de la Conservación en Galápagos", financiado por la Fundación Charles Darwin (2010-2012), y "Turismo de base local y sostenibilidad en Galápagos", financiado por el Plan Propio de Cooperación Internacional de la UPO (2011). En el segundo lo realizamos dentro de distintos proyectos que tienen como denominador común los procesos de patrimonialización y su efecto en la resiliencia socio-ecológica. 
BERKES, Fikret; HOLDING, Johan; FOLKE, Carl

2003 Navigating socio-ecological systems. Building resilience for complexity and change. Cambridge: Cambridge University Press.

BRINT, Steve

2001 "Gemeinschaft revisited”. Sociological Theory, 19, 1:1-23.

CARPENTER, Steve; WALKER, Brian; ANDERIES, J. Marty; et alt.

2001 "From metaphor to measurement. Resilience of what to what?". Ecosystems, 4:765-781.

\section{CEPAL}

1999 Centroamérica: Cambio institucional y desarrollo organizativo de las pequeñas unidades de producción rural. Puerto España: CEPAL. http://www.eclac.org/publicaciones/xml/8/9598/1406.pdf.

CORDERO ULATE, Allen

2006 "Territorio, economía y sociedad en Quepos-Manuel Antonio", en Nuevos ejes de acumulación y naturaleza. El caso del turismo. Buenos Aires: CLACSO, 133-156.

DAVIDSON-HUNT, Iain J.; BERKES, Fikret

2003 "Nature and society through the lens of resilience: toward and human-in-ecosystem perspective", en F. Berkes, J. Holding y C. Folke (eds.), Navigating socio-ecological systems. Building resilience for complexity and change. Cambridge: Cambridge University Press, 53-82.

DESCOLA, Philippe

2005 Par-delà nature et culture. Paris: Gallimard.

ESCALERA REYES, Javier

2008 "Turismo, espacios 'naturales' y resiliencia socio-ecológica", en Actas del II Congreso Latinoamericano de Antropología. San José, Costa Rica, 757-767.

2011 "Public Participation, Socio-ecological Resilience and Environmental Restoration: Lessons from two contrasting cases from Andalusia and Costa Rica”, en D. Egan, E. E. Hjerpe y J. Abrams (eds.), Human Dimensions of Ecological Restoration. Integrating Science, nature, and Culture. Washington: Island Press.

ESCOBAR, Arturo

1998 "Whose knowledge? Whose nature? Biodiversity conservation and the political ecology of social movements". Journal of Political Ecology, 5: 53-82.

1999 "After nature. Steps to an antiessentialist political ecology". Current Anthropology, 40:1-30.

FOLKE, Carl

2003 "Social-ecological resilience and behavioural responses", en A. Biel, B. Hansson, y M. Mårtensson (eds.), Individual and Structural Determinants of Environmental Practice. London: Ashgate Publishers, 226-287. 
FOLKE, Carl; CARPENTER, Steve; ELMQUIST, Thomas; et alt.

2002 "Resilience and sustainable development: building adaptative capacity in a world of transformations". Ambio 312, 5: 437-440.

GEERTZ, Clifford

1963 Agriculture involution: the process of ecological change in Indonesia. Berkeley: Univesity of California Press.

GERTLER, Michael

2001 "Pathways to Sustainable Resource Management: Coopesilencio", en Rural Cooperatives and Sustainable Development. Saskatoon: Centre for the Study of Co-operatives, 21-23.

HOLLING, Crawford Staley

1973 "Resilience and Stability of Ecologycal Systems". Annual Review of Ecology and Systematics, $4: 1-23$.

2001 "Understanding the complexity of economic, ecological and social systems". Ecosystems, 4: 390-405.

HONBORG, Alf

2001 "La ecología como semiótica. Esbozo de un paradigma contextualista para la ecología humana", en P. Descola y G. Pálson (coords.), Naturaleza y Sociedad, perspectivas antropológicas. Mexico: Siglo XXI, 80-100.

INGOLD, Tim

2000 The perception of the environment. London: Routledge.

LATOUR, Bruno

1991 Nous n'vons jamais été modernes: Essai d'anthropologie symétrique. Paris: La Découverte.

MARÍN HERNÁNDEZ, Juan José

1999 "La creación de Coopesilencio y la contribución de las mujeres campesinas en su edificación”. Diálogos: Revista electrónica de historia, 1, 1: 1-9.

MORAN, Emilio

1990 "Ecosystem Ecology in biology and anthropology: a critical assessment", en E. Moran (ed.), The ecosystem approach in Anthropology. Ann Arbor: University of Michigan Press, 41-72.

NORGAARD, Richard

1994 Development Betrayed. The end of progress and a coevolutionary revisioning of the future. New York: Routledge.

OSTROM, Elinor

2009 "A general framework for analyzing sustainability of socio-ecological systems". Science, 325: 419-422. 
PÁLSSON, Gísli

1996 "Human-environmental relations: orientalism, paternalism and communalism", en P. Descola y G. Palsson (ed.), Nature and Society: Anthropological Perspectives. London: Routledge, 63-81.

RAFFESTIN, Claude

1996 "Penser, classer, utiliser la nature", en M. Gonseth, J. Hainard, y R. Kaehr (eds.), Natures en tête. Neuchâtel: Musée d'ethnographie, 711-84.

RAPPAPORT, Roy A.

1967 "Ritual regulation of environtmental relations among a New Guinea people". Ethnology, 6, 1: 17-30.

1968 Pigs for the ancestors: ritual in the ecology of a New Guinea people. Long Grove: Waveland.

1990 "Ecosystems, populations and people", en E. Moran (ed.), The ecosystem approach in Anthropology. Ann Arbor: University of Michigan Press, 3-40.

RUIZ BALLESTEROS, Esteban

2007 "Del machete a la lengua'. Agua Blanca y la apropiación en el turismo comunitario", en E. Ruiz y D. Solis (coords.), Turismo comunitario en Ecuador. Quito: Abya-Yala, 227-314.

2009 Agua Blanca. Comunidad y turismo en el Pacifico ecuatorial. Quito: Abya-Yala.

2011 "Socio-ecological resilience and community-based tourism. An approach from Agua Blanca, Ecuador”. Tourism Management, 32: 655-666.

RUIZ BALLESTEROS, Esteban; HERNANDEZ, Macarena

2010 "Tourism that empowers? Commodification and appropriation in Ecuador's turismo comunitario". Critique of Anthropology, 30, 2: 201-229.

RUIZ BALLESTEROS, Esteban; HERNÁNDEZ, Macarena; COCA, Agustín; et alt.

2008 "Turismo comunitario en Ecuador. Comprendiendo el community-based tourism desde la comunidad". Pasos. Revista de turismo y patrimonio cultural, 6, 3: 399-418.

SCHEFFER, Marten

2009 Critical transitions in Nature and Society. Princeton: Princeton University Press.

SCOONES, Ian

1999 "New Ecology and the Social Science: What Prospects for a frutiful engagement?". Annual Review of Anthropology, 28: 479-507.

SOBRADO, Miguel

1998 "Coopesilencio: A un cuarto de siglo", en V. Barrantes (ed.), La construcción de un sueño. San José de Costa Rica: FELOPA-Universidad Nacional de Costa Rica, 125-147.

STEWART, Julian H.

1955 Theory of culture change: the methodology of multilinear evolution. Urbana: University of Illinois Press. 
TURNER, Nancy J.; DAVIDSON-HUNT, Iaian J.; O'FLAGHERTY, Michael

2003 "Living on the edge: ecological and cultural edges as sources of diversity for socioecological resilience". Human Ecology, 31, 3: 439-461.

VAYDA, Andrew P.; MCCAY, Bonnie J.

1975 "New directions in ecology and ecological anthropology". Annual Review of Anthropology, 4: 293-306.

WALKER, Brian H.; SALT, David

2006 Resilience Thinking: Sustaining Ecosystems and People in a Changing World. Washington: Island Press.

WALSH, John; HIGH, Steven

1999 "Rethinking the concept of community". Social History, 32, 64: 255-273.

WILSON, Alexander

1992 The Culture of Nature. Cambridge: Blackwell. 